\title{
ZETA FUNCTIONS AND ASYMPTOTIC ADDITIVE BASES WITH SOME UNUSUAL SETS OF PRIMES
}

\author{
WILLIAM D. BANKS
}

Aвstract. Fix $\delta \in(0,1], \sigma_{0} \in[0,1)$ and a real-valued function $\varepsilon(x)$ for which
$\lim _{x \rightarrow \infty} \varepsilon(x) \leqslant 0$. For every set of primes $\mathcal{P}$ whose counting function $\pi_{\mathcal{P}}(x)$ satisfies an estimate of the form

$$
\pi_{\mathcal{P}}(x)=\delta \pi(x)+O\left(x^{\sigma_{0}+\varepsilon(x)}\right),
$$

we define a zeta function $\zeta_{\mathcal{P}}(s)$ that is closely related to the Riemann zeta function $\zeta(s)$. For $\sigma_{0} \leqslant \frac{1}{2}$, we show that the Riemann hypothesis is equivalent to the non-vanishing of $\zeta_{\mathcal{P}}(s)$ in the region $\left\{\sigma>\frac{1}{2}\right\}$.

For every set of primes $\mathcal{P}$ that contains the prime 2 and whose counting function satisfies an estimate of the form

$$
\pi_{\mathcal{P}}(x)=\delta \pi(x)+O\left((\log \log x)^{\varepsilon(x)}\right),
$$

we show that $\mathcal{P}$ is an exact asymptotic additive basis for $\mathbb{N}$, i.e., for some integer $h=h(\mathcal{P})>0$ the sumset $h \mathcal{P}$ contains all but finitely many natural numbers. For example, an exact asymptotic additive basis for $\mathbb{N}$ is provided by the set

$$
\{2,547,1229,1993,2749,3581,4421,5281 \ldots\},
$$

which consists of 2 and every hundredth prime thereafter.

\section{INTRODUCTION AND STATEMENT OF RESULTS}

Let $\mathbb{N}$ denote the set of positive integers and $\mathbb{P}$ the set of prime numbers. Denote by $\pi(x)$ the prime counting function

$$
\pi(x):=\#\{p \leqslant x: p \in \mathbb{P}\},
$$

and for any given set of primes $\mathcal{P}$, put

$$
\pi_{\mathcal{P}}(x):=\#\{p \leqslant x: p \in \mathcal{P}\} .
$$

Given $\delta \in(0,1], \sigma_{0} \in[0,1)$ and a real function $\varepsilon(x)$ such that $\varlimsup_{x \rightarrow \infty} \varepsilon(x) \leqslant 0$, let $\mathscr{A}\left(\delta, \sigma_{0}, \varepsilon\right)$ denote the class consisting of sets $\mathcal{P} \subseteq \mathbb{P}$ for which one has an estimate of the form

$$
\pi_{\mathcal{P}}(x)=\delta \pi(x)+O\left(x^{\sigma_{0}+\varepsilon(x)}\right),
$$

where the implied constant may depend on $\mathcal{P}$. Let $\mathscr{B}(\delta, \varepsilon)$ denote the class consisting of sets $\mathcal{P} \subseteq \mathbb{P}$ such that

$$
\pi_{\mathcal{P}}(x)=\delta \pi(x)+O\left((\log \log x)^{\varepsilon(x)}\right),
$$

Date: March 27, 2018. 
where again the implied constant may depend on $\mathcal{P}$. The aim of this paper is to state some general results that hold true for all sets in $\mathscr{A}\left(\delta, \sigma_{0}, \varepsilon\right)$, or for all sets in $\mathscr{B}(\delta, \varepsilon)$. We also give examples of sets $\mathcal{P}$ in these classes, to which our general results can be applied.

1.1. Analogues of the Riemann zeta function. The Riemann zeta function is defined in the half-plane $\{s=\sigma+i t \in \mathbb{C}: \sigma>1\}$ by two equivalent expressions, namely

$$
\zeta(s):=\sum_{n \in \mathbb{N}} n^{-s}=\prod_{p \in \mathbb{P}}\left(1-p^{-s}\right)^{-1} \quad(\sigma>1) .
$$

In the extraordinary memoir of Riemann [19] it is shown that $\zeta(s)$ extends to a meromorphic function on the complex plane, its only singularity being a simple pole at $s=1$, and that it satisfies a functional equation relating its values at $s$ and $1-s$. The Riemann hypothesis $(\mathrm{RH})$ asserts that every non-real zero of $\zeta(s)$ lies on the critical line $\left\{\sigma=\frac{1}{2}\right\}$.

Although the function $\zeta(s)$ incorporates all of the primes into its definition, in this paper we observe that certain thin subsets of the primes also give rise to functions that are strikingly similar to $\zeta(s)$.

Theorem 1.1. For any set $\mathcal{P} \in \mathscr{A}\left(\delta, \sigma_{0}, \varepsilon\right)$, the function $\zeta_{\mathcal{P}}(s)$ defined by

$$
\zeta_{\mathcal{P}}(s):=\prod_{p \in \mathcal{P}}\left(1-p^{-s}\right)^{-1 / \delta} \quad(\sigma>1)
$$

extends to a meromorphic function on the region $\left\{\sigma>\sigma_{0}\right\}$, and there is a function $f_{\mathcal{P}}(s)$ which is analytic on $\left\{\sigma>\sigma_{0}\right\}$ and has the property that

$$
\zeta_{\mathcal{P}}(s)=\zeta(s) \exp \left(f_{\mathcal{P}}(s)\right) \quad\left(\sigma>\sigma_{0}\right) .
$$

This is proved in $\S 2$ below.

The following corollary is clear in view of (1.3); it shows that the truth of the Riemann hypothesis depends only on the distributional properties of certain (potentially thin) sets of primes.

Corollary 1.2. If $\mathcal{P} \in \mathscr{A}\left(\delta, \sigma_{0}, \varepsilon\right)$ and $\sigma_{0} \leqslant \frac{1}{2}$, then the Riemann hypothesis is true if and only if $\zeta_{\mathcal{P}}(s) \neq 0$ in the half-plane $\left\{\sigma>\frac{1}{2}\right\}$.

Similarly, for every nontrivial primitive Dirichlet character $\chi$, the Dirichlet $L$-function $L(s, \chi)$, which is initially defined by

$$
L(s, \chi):=\sum_{n \in \mathbb{N}} \chi(n) n^{-s}=\prod_{p \in \mathbb{P}}\left(1-\chi(p) p^{-s}\right)^{-1} \quad(\sigma>1),
$$

extends to an entire function on the complex plane and satisfies a functional equation relating its values at $s$ and $1-s$. The generalized Riemann hypothesis (GRH) asserts that every non-real zero of $L(s, \chi)$ lies on the critical line. 
The following result provides (in some cases) an analogue of Theorem 1.1. It is proved only for quadratic Dirichlet characters $\chi$. For any such character, let us denote

$$
\pi^{-}(x ; \chi):=\#\{p \leqslant x: p \in \mathbb{P} \text { and } \chi(p)=-1\},
$$

and for a given set of primes $\mathcal{P}$, put

$$
\pi_{\mathcal{P}}^{-}(x ; \chi):=\#\{p \leqslant x: p \in \mathcal{P} \text { and } \chi(p)=-1\} .
$$

Theorem 1.3. Fix $\mathcal{P} \in \mathscr{A}\left(\delta, \sigma_{0}, \varepsilon\right)$. Let $\chi$ be a primitive quadratic Dirichlet character, and suppose that

$$
\pi_{\mathcal{P}}^{-}(x ; \chi)=\rho \pi^{-}(x ; \chi)+O\left(x^{\sigma_{0}+\varepsilon(x)}\right),
$$

where $\rho \in(0,1]$. Suppose further that $\rho / \delta=A / B$ for two positive integers $A, B$. Then, the function $L_{\mathcal{P}}(s, \chi)$ defined by

$$
L_{\mathcal{P}}(s, \chi):=\prod_{p \in \mathcal{P}}\left(1-\chi(p) p^{-s}\right)^{-1 / \delta} \quad(\sigma>1)
$$

extends to a meromorphic function on the region $\left\{\sigma>\sigma_{0}\right\}$, and there is a function $f_{\mathcal{P}}(s, \chi)$ which is analytic on $\left\{\sigma>\sigma_{0}\right\}$ and has the property that

$$
\zeta(s)^{B} L_{\mathcal{P}}(s, \chi)^{B}=\zeta(s)^{A} L(s, \chi)^{A} \exp \left(f_{\mathcal{P}}(s, \chi)\right) \quad\left(\sigma>\sigma_{0}\right) .
$$

This is proved in $\S 3$ below.

1.2. Remarks. If one assumes that $\varepsilon(x)$ is such that the integral $\int_{1}^{\infty} x^{\varepsilon(x)-1} d x$ converges (for example, $\varepsilon(x):=-2(\log \log 2 x) / \log x)$, then $\zeta_{\mathcal{P}}(s)$ and $f_{\mathcal{P}}(s)$ in Theorem 1.1 extend to continuous functions in the closed half-plane $\left\{\sigma \geqslant \sigma_{0}\right\}$, and the relation (1.3) persists throughout $\left\{\sigma \geqslant \sigma_{0}\right\}$. For such $\varepsilon(x)$ one can easily deduce the following omega result in the case that $\sigma_{0}=\frac{1}{2}$.

Corollary 1.4. Let $\kappa: \mathbb{P} \rightarrow\{ \pm 1\}$ be a function that satisfies the estimate

$$
\#\{\text { prime } p \leqslant x: \kappa(p)=-1\}=\frac{1}{2} \pi(x)+O\left(x^{1 / 2+\varepsilon(x)}\right) .
$$

Then, for any primitive quadratic Dirichlet character $\chi$ we have

$$
\#\{\text { prime } p \leqslant x: \chi(p)=\kappa(p)\}=\Omega\left(x^{1 / 2+\varepsilon(x)}\right) .
$$

However, a stronger (and considerably more general) result has been obtained by Kisilevsky and Rubinstein [12]. Their work lies much deeper and utilizes explicit information about zeros of $L$-functions.

1.3. Examples. Here, we illustrate the results stated in $\S 1.1$ with some special sets of primes.

Let $p_{n}$ denote the $n$th smallest prime number for each positive integer $n$. Note that $n=\pi\left(p_{n}\right)$, thus $\pi(p)$ is the index associated to any given prime $p$. Let $\mathbb{P}_{k, b}$ denote the set of primes whose index lies in a fixed arithmetic progression 
$b \bmod k$; that is,

$$
\mathbb{P}_{k, b}:=\{p \in \mathbb{P}: \pi(p) \equiv b \bmod k\} .
$$

Let $\mathcal{P}:=\mathbb{P}_{k, b}$. Since $p_{n} \leqslant x$ if and only if $n \leqslant \pi(x)$, we have

$$
\pi_{\mathcal{P}}(x)=\#\{n \leqslant \pi(x): n \equiv b \bmod k\}=\left\lfloor\frac{\pi(x)-b}{k}\right\rfloor=\frac{1}{k} \pi(x)+O(1),
$$

where $\lfloor\cdot\rfloor$ is the floor function; this shows that (1.1) holds with $\delta=\frac{1}{k}, \sigma_{0}=0$, and $\varepsilon(x) \equiv 0$; in other words, $\mathbb{P}_{k, b} \in \mathscr{A}\left(\frac{1}{k}, 0,0\right)$. Applying Theorem 1.1 and Corollary 1.2 we immediately deduce the following.

Corollary 1.5. The function

$$
\zeta_{k, b}(s):=\prod_{p \in \mathbb{P}_{k, b}}\left(1-p^{-s}\right)^{-k} \quad(\sigma>1) .
$$

extends to a meromorphic function on the region $\{\sigma>0\}$, and there is a function $f_{k, b}(s)$ which is analytic on $\{\sigma>0\}$ and has the property that

$$
\zeta_{k, b}(s)=\zeta(s) \exp \left(f_{k, b}(s)\right) \quad(\sigma>0)
$$

Consequently, the Riemann hypothesis is true if and only if $\zeta_{k, b}(s) \neq 0$ in $\left\{\sigma>\frac{1}{2}\right\}$.

This shows that much analytic information about the Riemann zeta function (in particular, the location of the nontrivial zeros) is captured by a set of primes of relative density $\frac{1}{k}$.

More generally, for fixed $\kappa, \lambda \in \mathbb{R}$ with $\kappa \geqslant 1$, let $\mathcal{B}_{\kappa, \lambda}$ be the non-homogeneous Beatty sequence defined by

$$
\mathcal{B}_{\kappa, \lambda}:=\{n \in \mathbb{N}: n=\lfloor\kappa m+\lambda\rfloor \text { for some } m \in \mathbb{Z}\} .
$$

Beatty sequences appear in a variety of mathematical settings; the arithmetic properties of these sequences have been extensively explored in the literature. Let $\mathbb{P}_{\kappa, \lambda}$ denote the set of primes whose index lies in $\mathcal{B}_{\kappa, \lambda}$; that is,

$$
\mathbb{P}_{\kappa, \lambda}:=\left\{p \in \mathbb{P}: \pi(p) \in \mathcal{B}_{\kappa, \lambda}\right\} .
$$

As with (1.5) above, the estimate

$$
\pi_{\mathcal{P}}(x)=\frac{1}{\kappa} \pi(x)+O(1)
$$

is immediate; therefore, $\mathbb{P}_{\kappa, \lambda} \in \mathscr{A}\left(\frac{1}{\kappa}, 0,0\right)$, and one obtains a natural extension of Corollary 1.5 with the function

$$
\zeta_{\kappa, \lambda}(s):=\prod_{p \in \mathbb{P}_{\kappa, \lambda}}\left(1-p^{-s}\right)^{-\kappa} .
$$

Next, let $\mathbf{X}:=\left\{\mathbf{X}_{p}: p \in \mathbb{P}\right\}$ be a set of independent random variables, where each variable is either +1 or -1 , with a $50 \%$ probability for either value. The 
law of the iterated logarithm (due to Khintchine [11]) asserts that

$$
\varlimsup_{x \rightarrow \infty}(\pi(x) \log \log \pi(x))^{-1 / 2} \sum_{p \leqslant x} \mathbf{X}_{p}=\sqrt{2} \quad \text { a.s. }
$$

and (replacing $\left\{\mathbf{X}_{p}\right\}$ with $\left\{-\mathbf{X}_{p}\right\}$ ) that

$$
\varliminf_{x \rightarrow \infty}(\pi(x) \log \log \pi(x))^{-1 / 2} \sum_{p \leqslant x} \mathbf{X}_{p}=-\sqrt{2} \quad \text { a.s. }
$$

where "a.s." stands for "almost surely" in the sense of probability theory. In particular, denoting

$$
\mathbb{P}_{\mathbf{X}}^{+}:=\left\{p \in \mathbb{P}: \mathbf{X}_{p}=+1\right\} \quad \text { and } \quad \mathbb{P}_{\mathbf{X}}^{-}:=\left\{p \in \mathbb{P}: \mathbf{X}_{p}=-1\right\}
$$

we have the (less precise) estimate

$$
\pi_{\mathbb{P}_{\mathbf{X}}^{+}}(x)-\pi_{\mathbb{P}_{\mathbf{X}}^{-}}(x)=\sum_{p \leqslant x} \mathbf{X}_{p}=O\left(x^{1 / 2}\right) \quad \text { a.s. }
$$

Since $\pi(x)=\pi_{\mathbb{P}_{\mathbf{X}}^{+}}(x)+\pi_{\mathbb{P}_{\mathbf{X}}^{-}}(x)$ we deduce that

$$
\pi_{\mathbb{P}_{\overline{\mathbf{X}}}^{ \pm}}(x)=\frac{1}{2} \pi(x)+O\left(x^{1 / 2}\right) \quad \text { a.s. }
$$

for either choice of the sign \pm . Taking $\mathcal{P}:=\mathbb{P}_{\bar{x}}^{ \pm}$we see that (1.1) holds a.s. with $\delta=\sigma_{0}=\frac{1}{2}$ and $\varepsilon(x) \equiv 0$; in other words, $\mathbb{P}_{\overline{\mathbf{X}}}^{ \pm} \in \mathscr{A}\left(\frac{1}{2}, \frac{1}{2}, 0\right)$ almost surely. In view of Theorem 1.1 and Corollary 1.2 we deduce the following.

Corollary 1.6. In the region $\{\sigma>1\}$, let

$$
\zeta_{\boldsymbol{X}}^{+}(s):=\prod_{\substack{p \in \mathbb{P} \\ \boldsymbol{X}_{p}=+1}}\left(1-p^{-s}\right)^{-2} \quad \text { and } \quad \zeta_{\boldsymbol{X}}^{-}(s):=\prod_{\substack{p \in \mathbb{P} \\ \boldsymbol{X}_{p}=-1}}\left(1-p^{-s}\right)^{-2}
$$

Then, almost surely, both functions $\zeta_{\bar{X}}^{ \pm}(s)$ extend to meromorphic functions on the region $\left\{\sigma>\frac{1}{2}\right\}$, and there are functions $f_{\bar{X}}^{+}(s)$ which are analytic on $\left\{\sigma>\frac{1}{2}\right\}$ and are such that

$$
\zeta_{\bar{X}}^{ \pm}(s)=\zeta(s) \exp \left(f_{\bar{X}}^{ \pm}(s)\right) \quad\left(\sigma>\frac{1}{2}\right) .
$$

The Riemann hypothesis is equivalent to the assertion that, almost surely, $\zeta_{\bar{X}}^{+}(s) \neq 0$ in $\left\{\sigma>\frac{1}{2}\right\}$ for either choice of the sign \pm .

In the region $\{\sigma>1\}$, let us now define

$$
L(s, \mathbf{X}):=\prod_{p \in \mathbb{P}}\left(1-\mathbf{X}_{p} p^{-s}\right)^{-1} .
$$

The next corollary reproduces a result that was first proved by Wintner [25] and laid the foundation for random multiplicative function theory; it asserts that the GRH almost surely holds for the "L-function" $L(s, \mathbf{X})$ (for more modern work in this direction, see $[3,4,7,8,13])$. 
Corollary 1.7. The function $L(s, \boldsymbol{X})$ almost surely extends to an analytic function without zeros in the region $\left\{\sigma>\frac{1}{2}\right\}$.

Indeed, using (1.6) and (1.8) we have

$$
\begin{aligned}
L(s, \mathbf{X})^{2} & =\prod_{p \in \mathbb{P}}\left(1-\mathbf{X}_{p} p^{-s}\right)^{-2} \\
& =\prod_{p \in \mathbb{P}_{\mathbf{X}}^{+}}\left(1+p^{-s}\right)^{-2} \prod_{p \in \mathbb{P}_{\mathbf{X}}^{-}}\left(1-p^{-s}\right)^{-2} \\
& =\prod_{p \in \mathbb{P}_{\mathbf{X}}^{+}}\left(1-p^{-2 s}\right)^{-2} \prod_{p \in \mathbb{P}_{\mathbf{X}}^{+}}\left(1-p^{-s}\right)^{2} \prod_{p \in \mathbb{P}_{\mathbf{X}}^{-}}\left(1-p^{-s}\right)^{-2} \\
& =\zeta_{\mathbf{X}}^{+}(2 s) \zeta_{\mathbf{X}}^{+}(s)^{-1} \zeta_{\mathbf{X}}^{-}(s),
\end{aligned}
$$

By Corollary 1.6 there are (almost surely) functions $f_{\mathbf{X}}^{ \pm}(s)$ which are analytic on $\left\{\sigma>\frac{1}{2}\right\}$ and satisfy (1.7); in particular, the relation

$$
L(s, \mathbf{X})^{2}=\zeta_{\mathbf{X}}^{+}(2 s) \zeta_{\mathbf{X}}^{+}(s)^{-1} \zeta_{\mathbf{X}}^{-}(s)=\zeta(2 s) \exp \left(f_{\mathbf{X}}^{+}(2 s)-f_{\mathbf{X}}^{+}(s)+f_{\mathbf{X}}^{-}(s)\right)
$$

holds in $\{\sigma>1\}$, and it provides the required analytic continuation of $L(s, \mathbf{X})$ to the region $\left\{\sigma>\frac{1}{2}\right\}$. Moreover, $L(s, \mathbf{X}) \neq 0$ in $\left\{\sigma>\frac{1}{2}\right\}$.

\subsection{Asymptotic additive bases.}

TheOREM 1.8. Every set $\mathcal{P} \in \mathscr{B}(\delta, \varepsilon)$ containing the prime 2 is an exact asymptotic additive basis for $\mathbb{N}$. In other words, there is an integer $h=h(\mathcal{P})>0$ such that the $h$-fold sumset

$$
h \mathcal{P}:=\underbrace{\mathcal{P}+\cdots+\mathcal{P}}_{h \text { copies }}
$$

contains all but finitely many natural numbers.

This is proved in $\S 4$ below. We remark that Sárközy [20] has shown that any set of primes $\mathcal{P}$ is an asymptotic additive basis for $\mathbb{N}$, and stronger quantitative versions have been obtained; see $[\mathbf{1 4}, \mathbf{1 5}, \mathbf{1 8}, \mathbf{2 1}]$. To show that every $\mathcal{P} \in \mathscr{B}(\delta, \varepsilon)$ containing 2 is an exact asymptotic additive basis, we use a result of Shiu [22] on strings of consecutive primes in an arithmetic progression; in principle, the methods of Green and Tao [5] could be used to prove Theorem 1.8 with $\mathscr{B}(\delta, \varepsilon)$ replaced with a rather more restricted class of prime sets.

\subsection{Examples. As in $\S 1.3$, we put}

$$
\mathbb{P}_{k, b}:=\{p \in \mathbb{P}: \pi(p) \equiv b \bmod k\} .
$$

We have already seen that

$$
\pi_{\mathcal{P}}(x)=\frac{1}{k} \pi(x)+O(1)
$$


holds with $\mathcal{P}:=\mathbb{P}_{k, b}$, and therefore $\mathbb{P}_{k, b} \in \mathscr{B}\left(\frac{1}{k}, 0\right)$. Since $2 \in \mathbb{P}_{k, b}$ if and only if $b=1$, the next corollary follows immediately from Theorem 1.8.

Corollary 1.9. For every $k \in \mathbb{N}$, the set $\mathbb{P}_{k, 1}$ is an exact asymptotic additive basis for $\mathbb{N}$. For all $b, k \in \mathbb{N}$, the set $\mathbb{P}_{k, b} \cup\{2\}$ is an exact asymptotic additive basis for $\mathbb{N}$.

For example, an exact asymptotic additive basis for $\mathbb{N}$ is provided by the set

$$
\mathbb{P}_{100,1}=\{2,547,1229,1993,2749,3581,4421,5281 \ldots\},
$$

which consists of 2 and every hundredth prime thereafter.

More generally, for the set $\mathbb{P}_{\kappa, \lambda}$ defined in $\S 1.3$, we have the following result.

Corollary 1.10. For any $\kappa, \lambda \in \mathbb{R}$ with $\kappa \geqslant 1$, the set $\mathbb{P}_{\kappa, \lambda} \cup\{2\}$ is an exact asymptotic additive basis for $\mathbb{N}$.

\section{Proof of Theorem 1.1}

Suppose first that $s \in \mathbb{C}$ with $\sigma>1$. From the Euler product representations of $\zeta_{\mathcal{P}}(s)$ and $\zeta(s)$ we see that the function

$$
f_{\mathcal{P}}(s):=\log \zeta_{\mathcal{P}}(s)-\log \zeta(s)
$$

can be written in the form

$$
f_{\mathcal{P}}(s)=\sum_{j \geqslant 1} j^{-1} f_{\mathcal{P}, j}(s)
$$

with

$$
f_{\mathcal{P}, j}(s):=\frac{1}{\delta} \sum_{p \in \mathcal{P}} p^{-j s}-\sum_{p \in \mathbb{P}} p^{-j s} \quad(j \geqslant 1) .
$$

To prove the theorem, it is enough to show that $f_{\mathcal{P}}(s)$ extends to an analytic function in $\left\{\sigma>\sigma_{1}\right\}$ for every real number $\sigma_{1}>\sigma_{0}$.

Let $\sigma_{1}$ be given. Noting that $\sigma_{1}>0$, let $N$ be a positive integer such that $\sigma_{1}>\frac{1}{N}$. It is easy to verify that

$$
\sum_{j>N} j^{-1} f_{\mathcal{P}, j}(s)
$$

extends to an analytic function in $\left\{\sigma>\frac{1}{N}\right\}$, hence also in $\left\{\sigma>\sigma_{1}\right\}$. Therefore, it remains to show that for any fixed $j \in[1, N], f_{\mathcal{P}, j}(s)$ extends to an analytic function in $\left\{\sigma>\sigma_{1}\right\}$.

Using (1.1) we have

$$
\frac{1}{\delta} \pi_{\mathcal{P}}(u)=\pi(u)+E(u) \quad(u \geqslant 1),
$$


where $E(u) \ll u^{\sigma_{0}+\varepsilon(u)}$, and therefore

$$
\begin{aligned}
\frac{1}{\delta} \sum_{p \in \mathcal{P}} p^{-j s} & =\frac{1}{\delta} \int_{1}^{\infty} u^{-j s} d \pi_{\mathcal{P}}(u)=\frac{j s}{\delta} \int_{1}^{\infty} u^{-j s-1} \pi_{\mathcal{P}}(u) d u \\
& =j s \int_{1}^{\infty} u^{-j s-1} \pi(u) d u+j s \int_{1}^{\infty} u^{-j s-1} E(u) d u \\
& =\sum_{p \in \mathbb{P}} p^{-j s}+j s \int_{1}^{\infty} u^{-j s-1} E(u) d u
\end{aligned}
$$

that is,

$$
f_{\mathcal{P}, j}(s)=j s \int_{1}^{\infty} u^{-j s-1} E(u) d u
$$

Since $E(u) \ll u^{\sigma_{0}+\varepsilon(u)}$, the latter integral converges absolutely in $\left\{\sigma>j^{-1} \sigma_{0}\right\}$, hence also in $\left\{\sigma>\sigma_{1}\right\}$, and the integral representation provides the required analytic extension of $f_{\mathcal{P}, j}(s)$ when $j \in[1, N]$.

\section{Proof of Theorem 1.3}

As in $\S 2$ we first assume that $s \in \mathbb{C}$ with $\sigma>1$ and define

$$
f_{\mathcal{P}}(s, \chi):=B \log \left(\zeta(s) L_{\mathcal{P}}(s, \chi)\right)-A \log (\zeta(s) L(s, \chi))=\sum_{j \geqslant 1} j^{-1} f_{\mathcal{P}, j}(s, \chi),
$$

where

$$
f_{\mathcal{P}, j}(s, \chi):=\frac{B}{\delta} \sum_{p \in \mathcal{P}} \chi(p)^{j} p^{-j s}-A \sum_{p \in \mathbb{P}} \chi(p)^{j} p^{-j s}+(B-A) \sum_{p \in \mathbb{P}} p^{-j s} \quad(j \geqslant 1) .
$$

As before, let $\sigma_{1}>\sigma_{0}$ be given, and let $N$ be a fixed positive integer such that $\sigma_{1}>\frac{1}{N}$. To prove the theorem, it is enough to show that for any fixed $j \in[1, N]$ the function $f_{\mathcal{P}, j}(s, \chi)$ has an analytic extension to the region $\left\{\sigma>\sigma_{1}\right\}$.

Put

$$
\begin{aligned}
& f_{1}(s):=\frac{B}{\delta} \sum_{\substack{p \in \mathcal{P} \\
\chi(p)=1}} p^{-j s}-A \sum_{\substack{p \in \mathbb{P} \\
\chi(p)=1}} p^{-j s}, \\
& f_{2}(s):=\frac{B}{\delta} \sum_{\substack{p \in \mathcal{P} \\
\chi(p)=-1}} p^{-j s}-A \sum_{\substack{p \in \mathbb{P} \\
\chi(p)=-1}} p^{-j s}, \\
& f_{3}(s):=\frac{B}{\delta} \sum_{\substack{p \in \mathcal{P} \\
p \mid q}} p^{-j s}-A \sum_{\substack{p \in \mathbb{P} \\
p \mid q}} p^{-j s}, \\
& f_{4}(s):=(B-A) \sum_{p \in \mathbb{P}} p^{-j s},
\end{aligned}
$$

where $q$ is the modulus of the character $\chi$. We have

$$
f_{1}(s)+f_{2}(s)+f_{3}(s)+f_{4}(s)=\frac{B}{\delta} \sum_{p \in \mathcal{P}} p^{-j s}-B \sum_{p \in \mathbb{P}} p^{-j s}=B f_{\mathcal{P}, j}(s),
$$


where $f_{\mathcal{P}, j}(s)$ is given by (2.1). Recall that in $\S 2$ we have shown that $f_{\mathcal{P}, j}(s)$ has an analytic extension to the region $\left\{\sigma>\sigma_{1}\right\}$; the same is also true of $f_{3}(s)$ (which is clearly entire). Now observe that

$$
f_{\mathcal{P}, j}(s, \chi)=f_{1}(s)+(-1)^{j} f_{2}(s)+f_{4}(s),
$$

and therefore

$$
f_{\mathcal{P}, j}(s, \chi)= \begin{cases}-f_{3}(s)+B f_{\mathcal{P}, j}(s) & \text { if } j \text { is even, } \\ 2 f_{2}(s)-f_{3}(s)+B f_{\mathcal{P}, j}(s) & \text { if } j \text { is odd. }\end{cases}
$$

To conclude the proof it remains to show that $f_{2}(s)$ extends analytically to the region $\left\{\sigma>\sigma_{1}\right\}$.

Since $\rho / \delta=A / B$ we have

$$
f_{2}(s)=\frac{A}{\rho} \sum_{\substack{p \in \mathcal{P} \\ \chi(p)=-1}} p^{-j s}-A \sum_{\substack{p \in \mathbb{P} \\ \chi(p)=-1}} p^{-j s} .
$$

Using (1.4) we can write

$$
\frac{A}{\rho} \pi_{\mathcal{P}}(u, \chi)=A \pi(u, \chi)+E(u),
$$

where $E(u) \ll u^{\sigma_{0}+\varepsilon(u)}$. Then

$$
\begin{aligned}
\frac{A}{\rho} \sum_{\substack{p \in \mathcal{P} \\
\chi(p)=-1}} p^{-j s} & =\frac{A}{\rho} \int_{1}^{\infty} u^{-j s} d \pi_{\mathcal{P}}^{-}(u, \chi)=\frac{j s A}{\rho} \int_{1}^{\infty} u^{-j s-1} \pi_{\mathcal{P}}^{-}(u, \chi) d u \\
& =j s A \int_{1}^{\infty} u^{-j s-1} \pi^{-}(u, \chi) d u+j s \int_{1}^{\infty} u^{-j s-1} E(u) d u \\
& =A \sum_{\substack{p \in \mathbb{P} \\
\chi(p)=1}} p^{-j s}+j s \int_{1}^{\infty} u^{-j s-1} E(u) d u
\end{aligned}
$$

in other words,

$$
f_{2}(s)=j s \int_{1}^{\infty} u^{-j s-1} E(u) d u .
$$

Since $E(u) \ll u^{\sigma_{0}+\varepsilon(u)}$, the integral representation yields the desired analytic continuation of $f_{2}(s)$ to $\left\{\sigma>\sigma_{1}\right\}$.

\section{Proof of Theorem 1.8}

For the proof of Theorem 1.8, we use the following result of Banks, Güloğlu and Vaughan [2, Theorem 1.2] (the proof of which relies a deep theorem of Kneser; see Halberstam and Roth [6, Chapter I, Theorem 18]).

Lemma 4.1. Let $\mathcal{P}$ be a set of prime numbers such that

$$
\varliminf_{x \rightarrow \infty} \frac{\pi_{\mathcal{P}}(x)}{x / \log x}>0 .
$$


Suppose that there is a number $s_{1}$ such that for all $s \geqslant s_{1}$ and $a, b \in \mathbb{N}$, the congruence

$$
p_{1}+\cdots+p_{s} \equiv a \bmod b
$$

has a solution with $p_{1}, \ldots, p_{s} \in \mathcal{P}$. Then, there is an integer $h=h(\mathcal{P})>0$ such that the $h$-fold sumset $h \mathcal{P}$ contains all but finitely many natural numbers.

We also use the following statement concerning consecutive primes in a given arithmetic progression, which is due to Shiu [22, Theorem 1]; see also Banks et al [1, Corollary 4], where a bounded gaps variant is obtained as a consequence of the Maynard-Tao theorem (see [16]).

LEMMA 4.2. Let $p_{n}$ denote the $n$th smallest prime number for each positive integer $n$. Fix $c, d \in \mathbb{N}$ with $\operatorname{gcd}(c, d)=1$. Then, there are infinitely many $r \in \mathbb{N}$ such that $p_{r+1} \equiv p_{r+2} \equiv \cdots \equiv p_{r+m(r)} \equiv c \bmod d$, where $m(r)$ is an integer-valued function satisfying the lower bound

$$
m(r) \gg\left(\frac{\log \log r \log \log \log \log r}{(\log \log \log r)^{2}}\right)^{1 / \phi(d)} .
$$

Here, $\phi(\cdot)$ is the Euler function.

We now make an important observation based on Lemma 4.2, which may be of independent interest.

Proposition 4.3. Fix $\mathcal{P} \in \mathscr{B}(\delta, \varepsilon)$. For all $c, d \in \mathbb{N}$ with $\operatorname{gcd}(c, d)=1$, the set $\mathcal{P}$ contains infinitely many primes in the arithmetic progression $c \bmod d$.

Proof. According to Lemma 4.2, there is an infinite set $\mathcal{S} \subseteq \mathbb{N}$ with the property that

$$
p_{r+1} \equiv p_{r+2} \equiv \cdots \equiv p_{r+m(r)} \equiv c \bmod d \quad(r \in \mathcal{S}),
$$

where $m(r)$ satisfies (4.1). Taking into account (1.2), we derive the following estimate for all $r \in \mathcal{S}$ :

$$
\begin{aligned}
\pi_{\mathcal{P}}\left(p_{r+m(r)}\right)-\pi_{\mathcal{P}}\left(p_{r}\right) & =\delta\left(\pi\left(p_{r+m(r)}\right)-\pi\left(p_{r}\right)\right)+O\left((\log \log r)^{\varepsilon_{r}}\right) \\
& =\delta m(r)+O\left((\log \log r)^{\varepsilon_{r}}\right)
\end{aligned}
$$

where $\varepsilon_{r}:=\varepsilon\left(p_{r+m(r)}\right)$ for each $r$, and the constant implied by the $O$-symbol depends only on $\mathcal{P}$. In view of (4.1) and the fact that $\varlimsup_{r \rightarrow \infty} \varepsilon_{r} \leqslant 0$, we have

$$
\pi_{\mathcal{P}}\left(p_{r+m(r)}\right)>\pi_{\mathcal{P}}\left(p_{r}\right) \quad\left(r \in \mathcal{S}, r \geqslant r_{0}\right) .
$$

For every sufficiently large $r \in \mathcal{S}$, by (4.3) it follows that $p_{r+j} \in \mathcal{P}$ for some $j$ in the range $1 \leqslant j \leqslant m(r)$, and by (4.2) we have $p_{r+j} \equiv c \bmod d$. Since $\mathcal{S}$ is infinite, the lemma follows. 
Using the Hardy-Littlewood circle method, Vinogradov [24] established his famous theorem that every sufficiently large odd integer is the sum of three prime numbers. Effective versions of Vinogradov's theorem have been given by several authors (see $[\mathbf{1 0}, \mathbf{1 7}, \mathbf{2 3}]$ and references therein), but for the purposes of the present paper we require only the following extension of Vinogradov's theorem, which is due to Haselgrove [9, Theorem A].

LEMMA 4.4. For any fixed $\theta \in\left(\frac{63}{64}, 1\right)$ there is a positive number $n_{0}(\theta)$ such that every odd integer $n \geqslant n_{0}(\theta)$ can be expressed as the sum of three primes

$$
n=p_{1}+p_{2}+p_{3}
$$

with $\left|p_{j}-\frac{1}{3} n\right|<n^{\theta}$ for each $j=1,2,3$.

The following statement is a simple consequence of Haselgrove's result.

Lemma 4.5. For every integer $s \geqslant 6$, there is an integer $N_{0}(s)$ with the property that every integer $N \geqslant N_{0}(s)$ can be expressed as a sum of primes

$$
N=\widetilde{p}_{1}+\cdots+\widetilde{p}_{s}
$$

with $\widetilde{p}_{j}=2$ or $\widetilde{p}_{j} \geqslant \frac{1}{12} N$ for $j=1, \ldots, s$.

Proof. Set $\theta:=\frac{99}{100}$. Since $\theta \in\left(\frac{63}{64}, 1\right)$, Lemma 4.4 shows that there is a positive number $n_{0}=n_{0}(\theta)$ such that every odd integer $n \geqslant n_{0}$ can be expressed as the sum of three primes, $n=p_{1}+p_{2}+p_{3}$, with $p_{j} \geqslant \frac{1}{4} n$ for each $j=1,2,3$.

Put $N_{1}(s):=n_{0}+2 s-6$, and let $N$ be an odd integer exceeding $N_{1}(s)$. Since $n:=N-2 s+6$ is an odd integer exceeding $n_{0}$, we can write $n=p_{1}+p_{2}+p_{3}$ as above. Consequently,

$$
N=p_{1}+p_{2}+p_{3}+\underbrace{2+\cdots+2}_{s-3 \text { copies }}
$$

where

$$
p_{j} \geqslant \frac{1}{4} n=\frac{1}{4}(N-2 s+6) \quad(j=1,2,3) .
$$

Replacing $N_{1}(s)$ by a larger number, if necessary, the bound $N>N_{1}(s)$ and (4.4) together imply that $p_{j} \geqslant \frac{1}{12} N$ for $j=1,2,3$.

Next, put $N_{2}(s):=3 n_{0}+6 s-36$, and let $N$ be an even integer exceeding $N_{2}(s)$. If $n_{0}$ is sufficiently large (which we can assume) then $N-6 s+36=n+n^{\prime}$ for some odd integers $n$ and $n^{\prime}$ that are both larger than $\max \left\{n_{0}, \frac{1}{3} N\right\}$. Therefore, writing $n=p_{1}+p_{2}+p_{3}$ and $n^{\prime}=p_{1}^{\prime}+p_{2}^{\prime}+p_{3}^{\prime}$ as above, we have

$$
N=p_{1}+p_{2}+p_{3}+p_{1}^{\prime}+p_{2}^{\prime}+p_{3}^{\prime}+\underbrace{2+\cdots+2}_{s-6 \text { copies }}
$$

where

$$
p_{j} \geqslant \frac{1}{4} n \geqslant \frac{1}{12} N \quad \text { and } \quad p_{j}^{\prime} \geqslant \frac{1}{4} n^{\prime} \geqslant \frac{1}{12} N \quad(j=1,2,3) .
$$


Taking $N_{0}(s):=\max \left\{N_{1}(s), N_{2}(s)\right\}$ we finish the proof.

Proof of Theorem 1.8. Fix a set $\mathcal{P} \in \mathscr{B}(\delta, \varepsilon)$ with $2 \in \mathcal{P}$. Since $\pi_{\mathcal{P}}(x)$ satisfies (1.2) the first condition of Lemma 4.1 is met, and it remains only to verify the second condition of Lemma 4.1.

Fix an arbitrary integer $s \geqslant 6$, and let $a, b \in \mathbb{N}$ be given. Replacing $a$ with a sufficiently large number in the progression $a \bmod b$, we can assume that $a \geqslant 24 b$. We can further assume that $a$ exceeds the number $N_{0}(s)$ described in the statement of Lemma 4.5. Therefore, $a$ can be expressed as a sum of primes $a=\tilde{p}_{1}+\cdots+\tilde{p}_{s}$, where for every $j=1, \ldots, s$ we have either $\tilde{p}_{j}=2$ or else

$$
\tilde{p}_{j} \geqslant \frac{1}{12} a \geqslant 2 b>b \text {. }
$$

In the latter case, it is clear that $\operatorname{gcd}\left(\tilde{p}_{j}, b\right)=1$, hence by Proposition 4.3 there is a prime $p_{j} \in \mathcal{P}$ such that

$$
p_{j} \equiv \widetilde{p}_{j} \bmod b \text {. }
$$

Since $2 \in \mathcal{P}$, we can put $p_{j}:=2$ whenever $\widetilde{p}_{j}=2$, obtaining (4.5) in this case as well. Summing the congruences (4.5) over $j=1, \ldots, s$ gives

$$
a=\tilde{p}_{1}+\cdots+\widetilde{p}_{s} \equiv p_{1}+\cdots+p_{s} \bmod b .
$$

This shows that the second condition of Lemma 4.1 is met, and the proof of Theorem 1.8 is complete.

\section{ACKNOWLEDGEMENTS}

The author wishes to thank Tristan Freiberg, Andrew Granville, Victor Guo and Stephen Montgomery-Smith for their helpful comments.

\section{REFERENCES}

[1] W. D. Banks, T. Freiberg and C. L. Turnage-Butterbaugh, "Consecutive primes in tuples," Acta Arith. 167 (2015), no. 3, 261-266.

[2] W. D. Banks, A. M. Güloğlu and R. C. Vaughan, "Waring's problem for Beatty sequences and a local to global principle," J. Théor. Nombres Bordeaux 26 (2014), no. 1, $1-16$.

[3] S. Chatterjee and K. Soundararajan, "Random multiplicative functions in short intervals," Int. Math. Res. Not. (2012), no. 3, 479-492.

[4] A. Granville and K. Soundararajan, "The distribution of values of $L\left(1, \chi_{d}\right)$," Geom. Funct. Anal. 13 (2003), no. 5, 992-1028.

[5] B. Green and T. Tao, "Linear equations in primes," Ann. of Math. (2) 171 (2010), no. 3, 1753-1850.

[6] H. Halberstam and K. F. Roth, Sequences. Second edition. Springer-Verlag, New YorkBerlin, 1983.

[7] A. J. Harper, "On the limit distributions of some sums of a random multiplicative function," J. Reine Angew. Math. 678 (2013), 95-124.

[8] A. J. Harper, A. Nikeghbali and M. Radziwill, "A note on Helson's conjecture on moments of random multiplicative functions," preprint, 2015. arXiv:1505.01443 
[9] C. B. Haselgrove, "Some theorems in the analytic theory of numbers," J. London Math. Soc. 26 (1951), 273-277.

[10] H. A. Helfgott, "Chebotarev sets," 2011. Preprint available on arXiv: arXiv: 1312.7748

[11] A. Khintchine, "Über einen Satz der Wahrscheinlichkeitsrechnung," Fund. Math 6 (1924), 9-20.

[12] H. Kisilevsky and M. O. Rubinstein, "Chebotarev sets," 2011. Preprint available on arXiv: arXiv: 1112.4945

[13] Y.-K. Lau, G. Tenenbaum and J. Wu, "On mean values of random multiplicative functions," Proc. Amer. Math. Soc. 141 (2013), no. 2, 409-420.

[14] H. Li and H. Pan, "A density version of Vinogradov's three primes theorem," Forum Math. 22 (2010), no. 4, 699-714.

[15] K. Matomäki, "Sums of positive density subsets of the primes," Acta Arith. 159 (2013), no. 3, 201-225.

[16] J. Maynard, "Small gaps between primes," Ann. of Math. (2) 181 (2015), no. 1, 383-413.

[17] O. Ramaré, “On Šnirel'man's constant," Ann. Scuola Norm. Sup. Pisa Cl. Sci. (4) 22 (1995), no. 4, 645-706.

[18] O. Ramaré and I. Z. Ruzsa, "Additive properties of dense subsets of sifted sequences," J. Théor. Nombres Bordeaux 13 (2001), no. 2, 559-581.

[19] B. Riemann, "Ueber die Anzahl der Primzahlen unter einer gegebenen Grösse," Monatsberichte der Berliner Akademie, 1859.

[20] A. Sárközy, “On finite addition theorems. Structure theory of set addition." Astérisque 258 (1999), nos. xi-xii, 109-127.

[21] X. Shao, "A density version of the Vinogradov three primes theorem," Duke Math. J. 163 (2014), no. 3, 489-512.

[22] D. K. L. Shiu, "Strings of congruent primes," J. London Math. Soc. (2) 61 (2000), no. 2, 359-373.

[23] T. Tao, "Every odd number greater than 1 is the sum of at most five primes," Math. Comp. 83 (2014), no. 286, 997-1038.

[24] I. M. Vinogradov, "Representation of an odd number as a sum of three primes," Comptes Rendus (Doklady) de l'Academy des Sciences de l'USSR 15 (1937), 191-294.

[25] A. Wintner, "Random factorizations and Riemann's hypothesis," Duke Math. J. 11 (1944), 267-275.

Department of Mathematics, University of Missouri, Columbia MO, USA.

E-mail address: bankswd@missouri.edu 